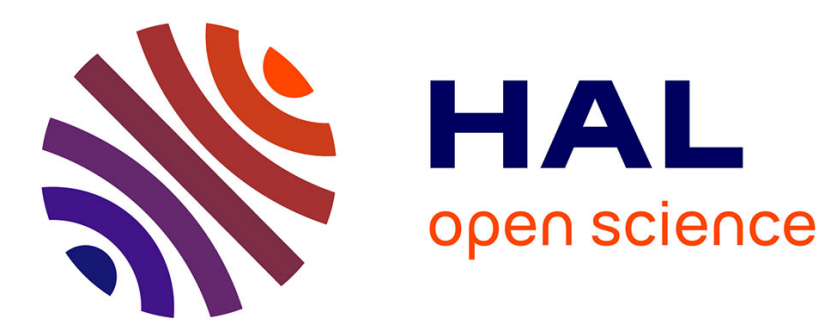

\title{
Link Quality Metrics in Large Scale Indoor Wireless Sensor Networks
}

\author{
Ana Bildea, Olivier Alphand, Franck Rousseau, Andrzej Duda
}

\section{To cite this version:}

Ana Bildea, Olivier Alphand, Franck Rousseau, Andrzej Duda. Link Quality Metrics in Large Scale Indoor Wireless Sensor Networks. Proceedings of the 24th IEEE International Symposium on Personal, Indoor and Mobile Radio Communications (PIMRC'13), 2013, London, United Kingdom. 10.1109/PIMRC.2013.6666451 . hal-01199098

\section{HAL Id: hal-01199098 \\ https://hal.science/hal-01199098}

Submitted on 28 Feb 2018

HAL is a multi-disciplinary open access archive for the deposit and dissemination of scientific research documents, whether they are published or not. The documents may come from teaching and research institutions in France or abroad, or from public or private research centers.
L'archive ouverte pluridisciplinaire HAL, est destinée au dépôt et à la diffusion de documents scientifiques de niveau recherche, publiés ou non, émanant des établissements d'enseignement et de recherche français ou étrangers, des laboratoires publics ou privés. 


\title{
Link Quality Metrics in Large Scale Indoor Wireless Sensor Networks
}

\author{
Ana Bildea, Olivier Alphand, Franck Rousseau, and Andrzej Duda \\ Grenoble Institute of Technology, CNRS Grenoble Informatics Laboratory UMR 5217, Grenoble, France \\ Email: \{firstname.lastname\}@imag.fr
}

\begin{abstract}
We report results of measurements on SensLAB, an indoor wireless sensor network testbed with a large number of nodes equipped with a CC1101 radio chip. We analyze RSSI and LQI to find the best way to discriminate good links from weak ones and in particular, to derive a metric that estimates the Packet Reception Ratio (PRR) the best. To obtain an estimator of PRR, we have fitted a Fermi-Dirac function to the scatter diagram of the average and standard variation of LQI. The function enables us to find PRR for a given level of LQI. We evaluate the estimator by computing PRR over a varying size window of transmissions and comparing with the estimator.
\end{abstract}

Keywords-Received Signal Strength indicator (RSSI), Link Quality Indicator (LQI), link and routing metrics, Wireless Sensor Networks (WSN), SensLAB

\section{INTRODUCTION}

Much research has considered the problem of characterizing the quality of wireless links to derive metrics for finding the best routes in wireless sensor networks. The transmission quality in terms of the Packet Reception Ratio (PRR) depends on the received signal strength, the level of interference, and the ability of the receiver to correctly decode transmitted information. The RSSI (Received Signal Strength Indicator) indicator provides an estimate of the signal energy at the receiver. Many studies revealed the importance of RSSI in the evaluation of link quality and analyzed the relation between RSSI and PRR.

One of the recent studies by Srinivasan et al. [1] showed that RSSI of CC2420 is a promising indicator when its value is above the sensitivity threshold. In particular, it allows to detect a threshold above which links present good PRR. Below the threshold, the PRR values largely vary and one RSSI value may correspond to several values of PRR. Nevertheless, RSSI has a low variance indicating that a RSSI value for one packet is a good estimator of an average RSSI value for consecutive packets. The authors also observed that LQI (Link Quality Indicator) presents a better correlation with PRR than RSSI, however, it needs to be averaged over many packets (about 120). LQI for CC1101 gives "an estimate of how easily a received signal can be demodulated by accumulating the magnitude of the error between ideal constellations and the received signal over the 64 symbols immediately following the sync word" [2].

Similar analysis by Rondinone et al. [3] led to the conclusion that neither RSSI nor LQI can be considered a successful link quality indicator since they do not sufficiently discriminate the level of PRR. They have proposed to use the product of PRR and the normalized average RSSI as a link quality indicator, which presents the advantage of distinguishing links with the same PRR, but with a different average RSSI. The drawback of this approach is that it requires the knowledge of PRR that we actually want to estimate based on the RSSI and LQI indicators.

In this paper, we report results of measurements on SensLAB [4], an indoor wireless sensor network testbed with a large number of nodes equipped with a CC1101 radio chip. We record the number of received packets and the values of RSSI and LQI for each transmission (no interference nor contention between nodes). We analyze the data to find the best way to discriminate good links from weak ones and in particular, derive a metric that estimates PRR the best. Recent work on routing protocols emphasized the importance of using stable metrics of link quality (one metric used with RPL is ETX that depends on PRR [5] and LOADng uses the number of weak links in a path as a metric [6]).

Our measurements confirm the previous results on the relationship between RSSI and PRR. Moreover, we use RSSI as an indicator of possible anomalous behavior of sensor nodes. To further characterize PRR in function of RSSI and LQI, we have looked for continuous distributions that fit the best the measured values of PRR. It comes out that RSSI is not a good discriminator of link categories, because the functions overlap. The average and standard variation of LQI better discriminate between the categories.

To obtain an estimator of PRR, we have fitted a FermiDirac function to the scatter diagram of the average and standard variation of LQI. The function enables us to find PRR for a given level of LQI. We evaluate the estimator by computing PRR over a varying size window of transmissions and comparing with the estimator.

\section{EXPERIMENTAL SET UP}

We have run experiments on the SensLAB platform [4] available in Strasbourg composed of 240 WSN430 nodes distributed across three trays at different heights. Each tray contains 80 nodes arranged in a regular grid (10x8) with a distance between contiguous nodes of about $1 \mathrm{~m}$. A node is composed of a MSP430F1611 CPU (48KB ROM, 10KB $\mathrm{RAM}$ ) and a CC1101 radio operating at $868 \mathrm{MHz}$. Its transmission power ranges between $-30 \mathrm{dBm}$ and $10 \mathrm{dBm}$, and the reception sensitivity is set to $-88 \mathrm{dBm}$.

In a single experiment, we use one tray at a time, i.e. 80 nodes. We observe the quality of transmission of a node that broadcasts a total of 5000 packets of 110 bytes every $0.5 \mathrm{~s}$. There is no other ongoing transmissions so there is no 
interference nor contention between nodes. When one node broadcasts its packet, the other 79 nodes are active and ready to receive - they log the values of LQI and RSSI of the received packet. The values are recorded for the correctly received packets with good CRC and also for those with incorrect CRC. As there is one sender at a time, we are able to relate the sender and the receiver of a packet even if the receiver cannot decode a packet.

The receiver nodes do not acknowledge frames and the MAC layer does not retransmit frames in case of failed transmissions. After the experiment, we compute for each link: i) the average value of RSSI over all received packets, ii) the average value and the standard deviation of LQI, iii) the average value of the Packet Reception Ratio (PRR) of each link as the proportion between the number of correctly received packets (correct CRC) to the total number of sent packets. The observed values of RSSI only slightly varied, so we have not analyzed the standard deviation of RSSI.

We assume that all nodes can potentially communicate with each other so that the number of unidirectional links is 6320 ( 80 sender nodes times 79 receiver nodes). We run the experiments with two levels of the transmission power: $0 \mathrm{dBm}$ and $-10 \mathrm{dBm}$. The bit rate is $60 \mathrm{~kb} / \mathrm{s}$ and nodes use the 2FSK modulation. Table I summarizes the parameters of the experiments.

TABLE I. EXPERIMENT PARAMETERS

\begin{tabular}{ll}
\hline Experiment area & $10 \mathrm{~m} \times 8 \mathrm{~m} \mathrm{x} 2 \mathrm{~m}$ \\
\hline Number of nodes & $3 \times 80$ \\
\hline Traffic type, interpacket interval & broadcast, $0.5 \mathrm{~s}$ \\
\hline Number of sent packets & 5000 \\
\hline Packet size & 110 bytes \\
\hline Transmission power & $0 \mathrm{dBm},-10 \mathrm{dBm}$ \\
\hline Topology & grid
\end{tabular}

\section{LINK CHARACTERIZATION}

\section{A. Categories of Link Quality}

We consider three main categories of link quality: good links with $P R R \geq 80 \%$, intermediate with the PRR $20 \% \leq$ $P R R<80 \%$, and bad ones with $0<P R R<20 \%$. Such categories appeared in previous studies [7], [8], but other thresholds are possible, e.g. 90\%-10\%.

Table II gives the proportion of links in each category (over all 6320 unidirectional links). In addition to the categories, we provide the proportion of the links with $P R R=0 \%$ (actually, no link between nodes).

TABLE II. PROPORTION OF LINKS IN EACH CATEGORY

\begin{tabular}{ccccc}
\hline Transmission power & good & intermediate & bad & $P R R=0 \%$ \\
\hline $\mathbf{0 d B m}$ & $49 \%$ & $8 \%$ & $10 \%$ & $33 \%$ \\
\hline $\mathbf{- 1 0 d B m}$ & $44 \%$ & $6 \%$ & $9 \%$ & $41 \%$ \\
\hline
\end{tabular}

We can observe that roughly half of links are good, a large number of links are bad and decreasing the transmission power to $-10 \mathrm{dBm}$ only slightly affects the proportion of good links. The number of intermediate links is fairly low.

\section{B. Analysis of RSSI}

We have started with the analysis of RSSI and its influence on PRR. Fig. 1 presents the scatter diagram of PRR vs. the average RSSI for all links (each point corresponds to a link with a given average RSSI and the observed PRR).

Some values (red crosses) lie in a region with high PRR and low RSSI values. It correspond to two nodes that generated anomalous RSSI samples. A deeper RSSI analysis enables us to detect nodes with hardware anomalies or bad calibration that result in unlikely values of RSSI. In the case of our testbed, there is a small proportion of anomalous nodes: 2 out of 80 . Thus, in the following, we eliminate the results from those nodes to take away the bias of unlikely RSSI values.

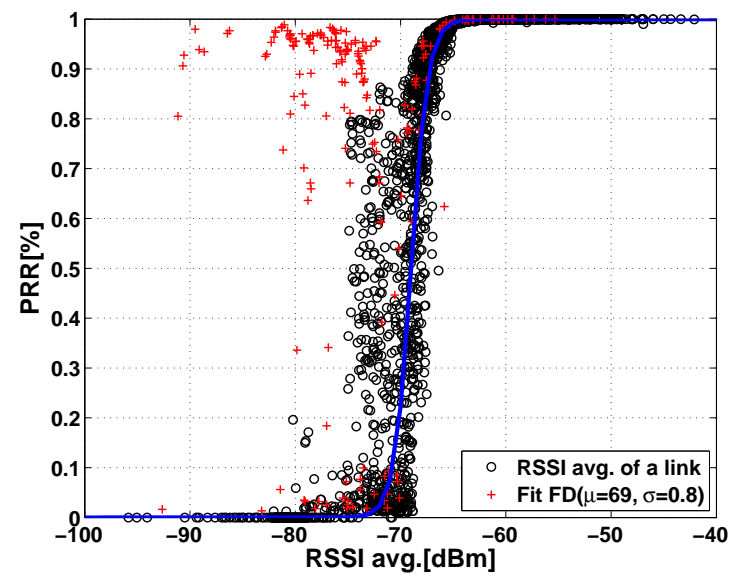

Fig. 1. Fitting of the scatter diagram with a Fermi-Dirac function.

The next step is to explore the dependence between RSSI and PRR. Fig. 1 also presents the fitting of the scatter diagram with a Fermi-Dirac function of the form $f(x)=$ $1 /\left(1+\exp \frac{-(\mu-x)}{\sigma}\right)$.

We have also averaged the values of RSSI for each value of PRR (at the step of $1 \%$ ) and found the fitting function presented in Fig. 2. We can see that the Fermi-Dirac function fits perfectly well PRR especially the values greater than $80 \%$.

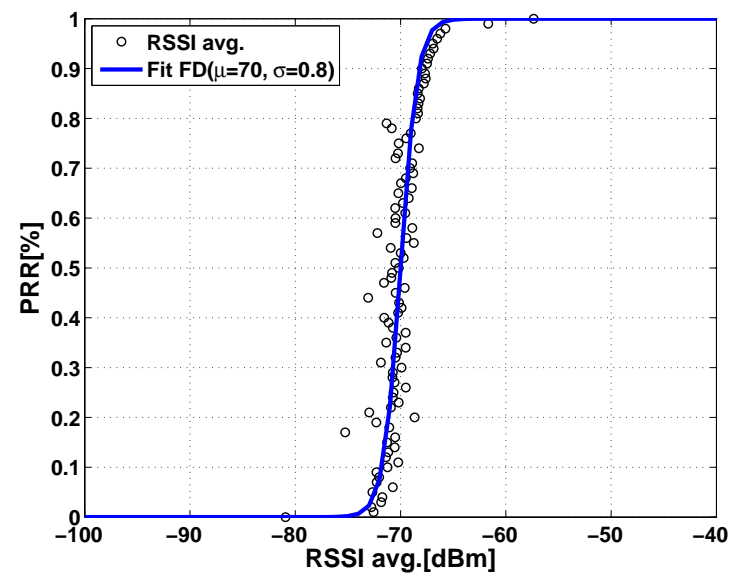

Fig. 2. Fitting of the averaged RSSI.

We can use the function to determine a cutting threshold over which the RSSI results in a PRR of good links.

\section{Fitting the Distributions of RSSI and LQI}

To further characterize PRR in function of RSSI and LQI, we have looked for continuous distributions that fit the best the 
measured values of PRR. The goal is to predict PRR or at least the link category based on the observed values of RSSI and LQI. We have considered as candidate distributions the most common fifty continuous distributions with a bounded, semiinfinite, variable support or unbounded. We have used Matlab as environment to find the best distribution fit for our data set. The distributions that fitted the best are the following:

- Log-Logistic distribution is defined by scale $\alpha$, shape $\beta$, and location $\gamma$. Its Cumulative Distribution Function $(\mathrm{CDF})$ is $F_{x ; \alpha, \beta, \gamma}=\left(1+\left(\beta /(x-\gamma)^{\alpha}\right)^{-1}\right.$. The Log-Logistic models the mid of the extreme values (highs and lows) of a data set of random variables.

- Johnson $S B$ distribution has a bounded support that fits a bounded distribution to known moments and has CDF of $F_{x}=\phi(\gamma+\delta \ln (z /(1-z)))$, where $\phi$ is the Laplace integral and $z$ is defined as $(x-\xi) / \lambda$. It is parametrized with shape $\gamma$, two scale parameters $\delta$ and $\lambda$, and location parameter $\xi$.

- Generalized Extreme Value is a distribution that models the maxima of the extreme values of a data set. It is parametrized by shape $\xi$, scale $\sigma$, and location $\mu$. Its $\mathrm{CDF}$ is $F(x ; \mu, \sigma, \xi)=\exp \left\{-\left[1+\xi\left(\frac{x-\mu}{\sigma}\right)\right]^{-1 / \xi}\right\}$.

- Beta distribution is defined on the interval $[0,1]$ with two positive continuous shape parameters $\alpha$ and $\beta$. Its $\mathrm{CDF}$ is $F(x ; \alpha, \beta)=B(x ; \alpha, \beta) / B(\alpha, \beta)=I_{x}(\alpha, \beta)$, where $B(x ; \alpha, \beta)$ is the incomplete beta function and $I_{x}(\alpha, \beta)$ is the regularized incomplete beta function. The incomplete beta function is the following: $B(x ; a, b)=\int_{0}^{x} t^{a-1}(1-t)^{b-1} d t$.

We have used three common statistical tests: KolmogorovSmirnov, $\chi^{2}$, and Anderson-Darling [9] to find the best distributions.

We first categorize the measured values into three categories (good, intermediate, and bad) and run fit tests on candidate distributions. To decrease the number of samples to handle, we average the measured values of LQI and RSSI for each link.

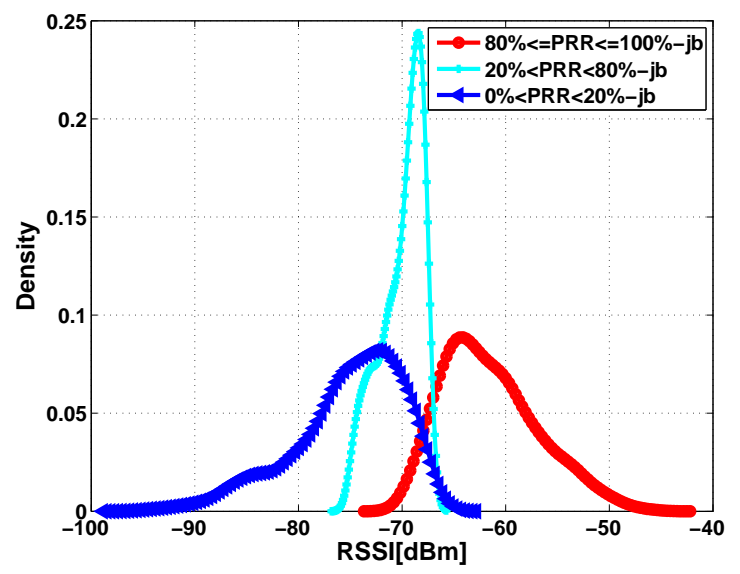

Fig. 3. Density functions fitting the average RSSI.

Figs. 3-5 show the best density functions for each category: Johnson SB for RSSI and Generalized Extreme Value as

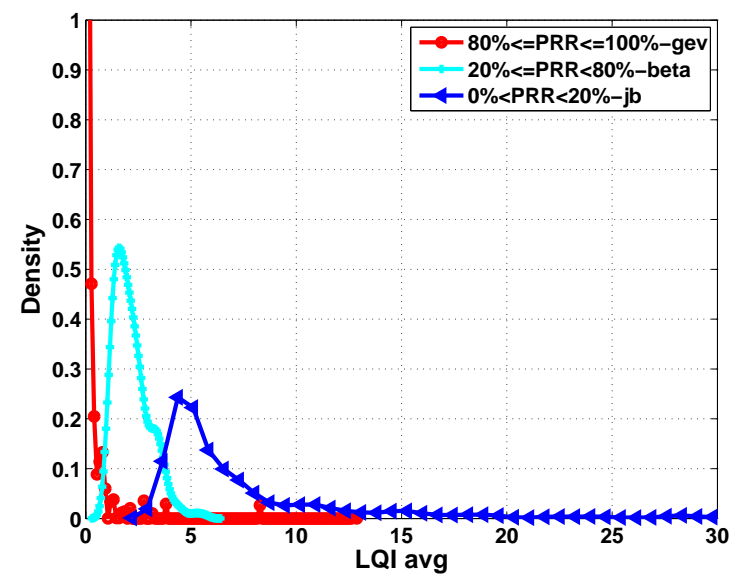

Fig. 4. Density functions fitting the average LQI.

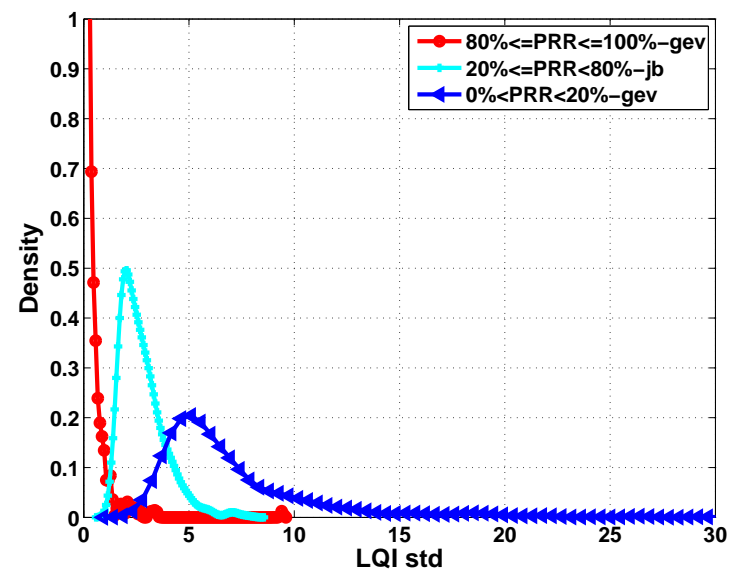

Fig. 5. Density functions fitting the standard deviation of LQI.

well as Beta, and Johnson SB for LQI. We can observe that RSSI is not a good discriminator of link categories, because the functions overlap. Even the bad category overlaps the category of good links. The average LQI better discriminates between the categories, especially it can distinguish good from intermediate links. We can also observe that the standard variation of LQI is also a good discriminator of the categories.

\section{Fitting PRR in Function of LQI}

We have applied a similar approach to LQI-fitting a Fermi-Dirac function to the scatter diagram of the average and standard variation of LQI (we use here the logarithmic scale for LQI). Figs. 6-7 present the corresponding result.

\section{E. Estimating PRR}

We want to find an estimator of PRR based on the measured values of RSSI and LQI. We have fitted PRR in function of RSSI, so possibly we could derive PRR from a given value of RSSI. However, using RSSI may lead to errors in the evaluation of PRR in the case of real networksthey experience contention between nodes and simultaneous transmissions. Concurrent transmissions may increase RSSI and decrease the probability of correct decoding leading to a lower PRR. In this case, the estimation based on RSSI would result in wrong values of PRR. So, we have decided to focus 


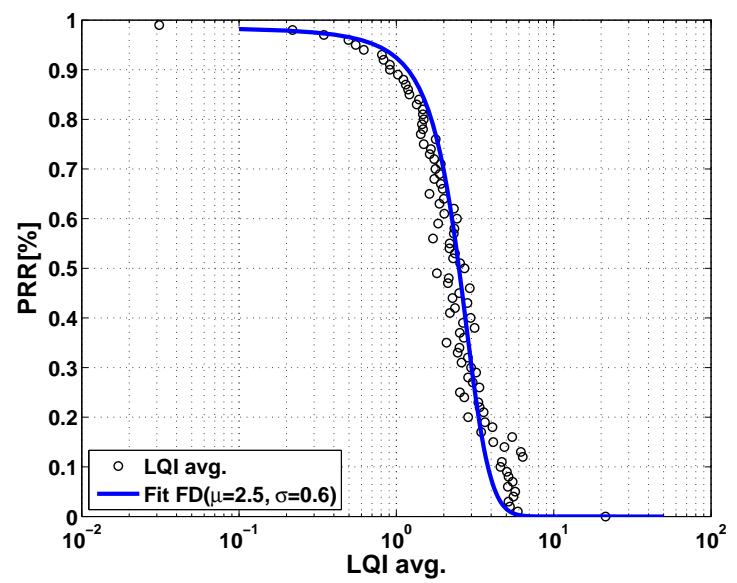

Fig. 6. Fitting LQI averaged over links.

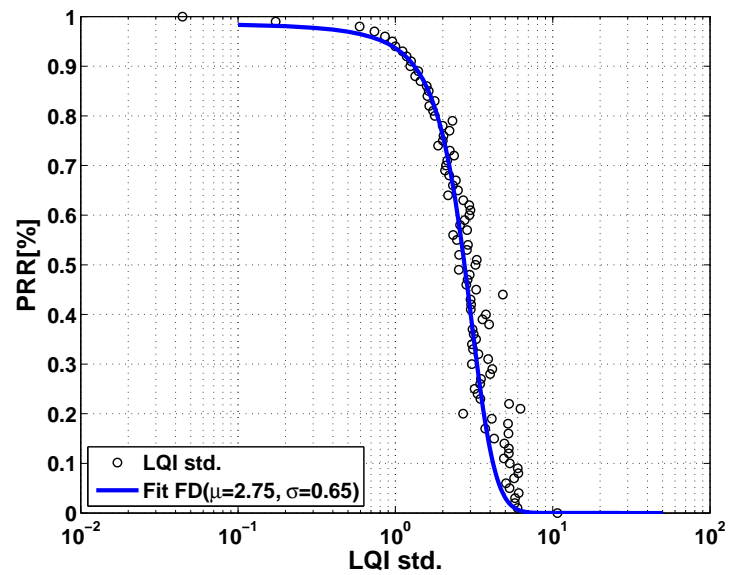

Fig. 7. Fitting the standard deviation of LQI averaged over links.

on LQI and analyze estimators based on its average value and standard deviation.

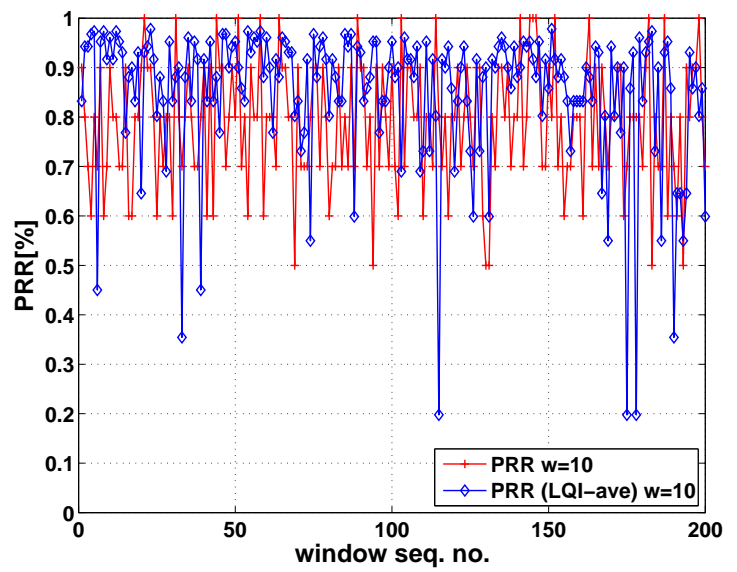

Fig. 8. Estimation of the PRR for a link with PRR $80 \%$ with $w=10$. PRR vs. LQI avg.

Fig. 8 shows the temporal behavior of PRR computed over a window of 10 transmissions and its estimator derived from the average LQI. We can observe a fairly good fit between the values.
We have tested several estimators based on the values computed on window $w: \overline{L Q I}$, average LQI, $\overline{S T D}$, the standard

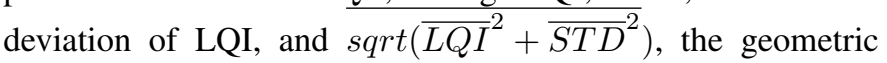
mean of the previous ones. We have also derived the estimator of PRR from a moving average of LQI:

$$
\operatorname{EWMA}(\alpha, n)=\operatorname{EWMA}(\alpha, n-1) * \alpha+(1-\alpha) * \mathrm{LQI}
$$

where $0<\alpha<1$. We have considered two values of the window $w=10,100$. Tables III-V present the precision of the estimators in terms of several standard error measures: $R^{2}$, MSE (Mean Square Error), RMSE (Root Mean Square Error). We can observe that for the short window, the estimation based on LQI results in an error of the order of $18 \%$ (RMSE). Considering larger windows reduces the error to 9\%. EWMA with $\alpha=0.9$ also results in a good precision.

\section{RELATED WORK}

Much research work considered the problem of characterizing the quality of wireless links in sensor networks. On a 60 mote indoor/outdoor testbed, Zhao et al. [10] showed the existence of spatial gray regions corresponding to high variation in packet reception. Woo et al. [11] observed good connectivity of links to nodes up to $3 \mathrm{~m}$ and a transitional (grey) zone between $3 \mathrm{~m}$ and $12 \mathrm{~m}$ with links exhibiting large variability. In another paper, the authors modeled packet loss with a binomial distribution and observed that good links are symmetric [12]. Other researchers confirmed the existence of three zones: the well connected, the transitional (grey), and the disconnected one [13], [14]. They reported that the links in the connected zone are stable contrary to the transitional zone.

As mentioned in the introduction, Srinivasan et al. [1] analyzed RSSI and LQI of CC2420. They highlighted the utility of RSSI in detecting links with good PRR and observed good correlation of LQI and PRR. Meier et al. [15] analyzed measurements of an indoor sensor network with CC2420 exhibiting high link quality variability and considered metrics derived from modeling the loss process as a Bernoulli process.

Tang et al. [16] evaluated the temporal and spatial link fluctuation in a factory environment and characterized channel variations. Bas et al. [17] demonstrated that the angle of the direction influences the correlation of the link quality with the distance. Rondinone et al. [3] analyzed the link quality in sensor networks and proposed to use as a link quality indicator the product of PRR and the normalized average RSSI.

Our analysis confirms the main findings of the previous work and provides new insights on the link quality metrics based on LQI.

\section{CONCLUSION}

In this paper, we have reported results of measurements of PRR, RSSI, and LQI on an indoor wireless sensor network testbed. We have analyzed RSSI and used it as an indicator of possible anomalous behavior of sensor nodes. To further characterize PRR in function of RSSI and LQI, we have looked for continuous distributions that fit the best the measured values of PRR. As RSSI is not a good discriminator of link categories, we have considered the average LQI and its standard variation. We have found the density functions that 
TABLE III. ERROR OF ESTIMATING PRR FOR A GOOD LINK (MEAN PRR OF 80\%).

\begin{tabular}{|c|c|c|c|c|c|c|}
\hline Estimator & $R^{2}, w=10$ & $R^{2}, w=100$ & MSE, $w=10$ & MSE, $w=100$ & RMSE, $w=10$ & RMSE, $w=100$ \\
\hline$\overline{\mathrm{LQI}}$ & 0.55 & 0.94 & 0.03 & 0.01 & 0.17 & 0.1 \\
\hline$\overline{\mathrm{STD}}$ & 0.75 & 0.99 & 0.06 & 0.06 & 0.24 & 0.24 \\
\hline${\overline{\operatorname{sqrt}}\left(\overline{\mathrm{LQI}}^{2}+\overline{\mathrm{STD}}^{2}\right)}_{\text {EWMA }_{\mathrm{LQI}}(\alpha=0.1)}(\alpha=0.55$ & 0.99 & 0.03 & 0.04 & 0.18 & 0.20 \\
\hline EWMA $_{\mathrm{LQI}}(\alpha=0.5)$ & 0.80 & 0.99 & 0.08 & 0.08 & 0.28 & 0.28 \\
\hline EWMA $_{\mathrm{LQI}}(\alpha=0.9)$ & 0.53 & 0.96 & 0.07 & 0.06 & 0.27 & 0.24 \\
\hline
\end{tabular}

TABLE IV. ERROR OF ESTIMATING PRR FOR AN INTERMEDIATE LINK (MEAN PRR OF 50\%).

\begin{tabular}{|c|c|c|c|c|c|c|}
\hline Estimator & $R^{2}, w=10$ & $R^{2}, w=100$ & MSE, $w=10$ & MSE, $w=100$ & RMSE, $w=10$ & RMSE, $w=100$ \\
\hline 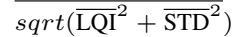 & 0.70 & 0.97 & 0.09 & 0.12 & 0.30 & 0.35 \\
\hline $\mathrm{EWMA}_{\mathrm{LQI}}(\alpha=0.5)$ & 0.72 & 0.97 & 0.15 & 0.13 & 0.39 & 0.36 \\
\hline $\mathrm{EWMA}_{\mathrm{LQI}}(\alpha=0.9)$ & 0.78 & 0.96 & 0.13 & 0.11 & 0.36 & 0.33 \\
\hline
\end{tabular}

TABLE V. ERROR OF ESTIMATING PRR FOR AN INTERMEDIATE LINK (MEAN PRR OF 20\%).

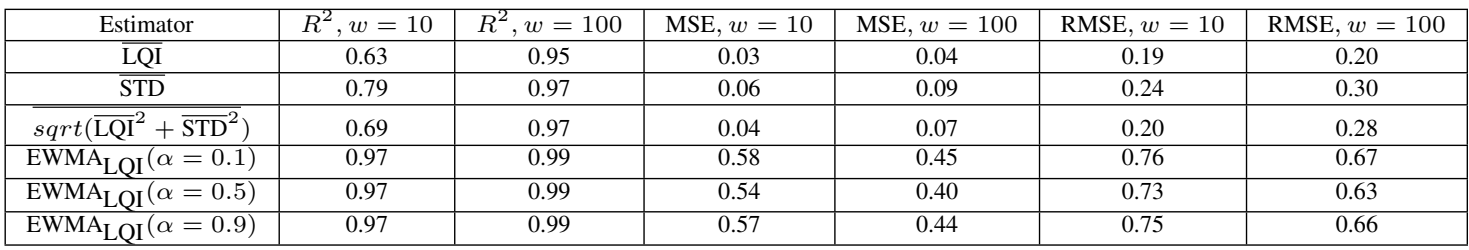

fit the observed values for three categories of links. To obtain an estimator of PRR, we have fitted a Fermi-Dirac function to the scatter diagram of the average and standard variation of LQI. The function enables us to find PRR for a given level of LQI. We have evaluated the estimator by computing PRR over a varying size window of transmissions and comparing with the estimator.

In the future, we plan to use the results of the link characterization in routing and validate the approach on the SensLAB testbed.

\section{ACKNOWLEDGEMENT}

This work was partially supported by the French National Research Agency (ANR) project ARESA2 under contract ANR-09-VERS-017, ANR project IRIS under contract ANR11-INFR-016, and the European Commission FP7 project CALIPSO under contract 288879.

\section{REFERENCES}

[1] K. Srinivasan and P. Levis, "RSSI is Under Appreciated," in Proc. of the Third Workshop on Embedded Networked Sensors (EmNets), 2006.

[2] TI, "Calculation and Usage of LQI and RSSI." [Online]. Available: \{http://e2e.ti.com/support/low_power_rf/w/design_ notes/calculation-and-usage-of-lqi-and-rssi.aspx $\}$

[3] M. Rondinone, J. Ansari, J. Riihijärvi, and P. Mähönen, "Designing a Reliable and Stable Link Quality Metric for Wireless Sensor Networks," in Proceedings of the Workshop on Real-World Wireless Sensor Networks, ser. REALWSN '08, 2008, pp. 6-10.

[4] C. Burin des Rosiers et al., "SensLAB: Very Large Scale Open Wireless Sensor Network Testbed," in Proc. 7th TridentCOM Conference, Shanghai, Chine, Apr. 2011.

[5] T. Winter, P. Thubert, A. Brandt, J. Hui, R. Kelsey, P. Levis, K. Pister R. Struik, J. Vasseur, and R. Alexander, "RPL: IPv6 Routing Protoco for Low power and Lossy Networks," IETF, RFC 6550, March 2012.
[6] T. Clausen, A. Colin de Verdiere, J. Yi, A. Niktash, Y. Igarashi, H. Satoh, U. Herberg, C. Lavenu, T. Lys, C. Perkins, and J. Dean, "The Lightweight On-demand Ad hoc Distance-vector Routing Protocol - Next Generation (LOADng)," IETF, Work in Progress draft-clausenlln-loadng-08, January 2013.

[7] T. Watteyne, S. Lanzisera, A. Mehta, and K. S. J. Pister, "Mitigating Multipath Fading through Channel Hopping in Wireless Sensor Networks," in Proc. of IEEE International Conference on Communications. Ieee, May 2010, pp. 1-5.

[8] K. Srinivasan, P. Dutta, A. Tavakoli, and P. Levis, "An Empirical Study of Low-Power Wireless," ACM Trans. Sen. Netw., vol. 6, no. 2, pp. 16:1-16:49, Mar. 2010.

[9] P. J. Huber, Robust Statistics. Wiley, 2004.

[10] J. Zhao and R. Govindan, "Understanding Packet Delivery Performance in Dense Wireless Sensor Networks," in Proc. SenSys, 2003, pp. 1-13.

[11] A. Woo, T. Tong, and D. Culler, "Taming the Underlying Challenges of Reliable Multihop Routing in Sensor Networks," in In SenSys. ACM Press, 2003, pp. 14-27.

[12] A. Woo and D. Culler, "Evaluation of Efficient Link Reliability Estimators for Low-Power Wireless Networks," EECS Department, University of California, Berkeley, Tech. Rep. UCB/CSD-03-1270, 2003.

[13] M. Zennaro, H. Ntareme, and A. Bagula, "Experimental Evaluation of Temporal and Energy Characteristics of an Outdoor Sensor Network," Proceedings of the Mobility '08 Conference, 2008.

[14] N. Baccour et al., "RadiaLE: A Framework for Designing and Assessing Link Quality Estimators in Wireless Sensor Networks," Ad Hoc Netw., vol. 9, no. 7, pp. 1165-1185, Sep. 2011.

[15] A. F. Meier, T. Rein, J. Beutel, and L. Thiele, "Coping with Unreliable Channels: Efficient Link Estimation for Low-Power Wireless Sensor Networks," in Proc. 5th Intl. Conf. Networked Sensing Systems (INSS 2008), Kanazawa, Japan, Jun 2008, pp. 19-26.

[16] L. Tang, K.-C. Wang, Y. Huang, and F. Gu, "Channel Characterization and Link Quality Assessment of IEEE 802.15.4-Compliant Radio for Factory Environments," IEEE Transactions on Industrial Informatics, vol. 3, no. 2, pp. $99-110$, May 2007.

[17] C. U. Bas and S. C. Ergen, "Spatio-Temporal Characteristics of Link Quality in Wireless Sensor Networks," in Proc. of WCNC, 2012, pp. 1152-1157. 